\title{
Review of Tm and Ho Materials; Spectroscopy and Lasers
}

\author{
*B.M. Walsh \\ NASA Langley Research Center, Hampton, VA 23681 \\ * brian.m.walsh@nasa.gov
}

\begin{abstract}
A review of Tm and Ho materials is presented, covering some fundamental aspects on the spectroscopy and laser dynamics in both single and co-doped systems. Following an introduction to $2-\mu \mathrm{m}$ lasers, applications and historical development, the physics of quasi-four level lasers, energy transfer and modeling are discussed in some detail. Recent developments in using Tm lasers to pump Ho lasers are discussed, and seen to offer some advantages over conventional Tm:Ho lasers. This article is not intended as a complete review, but as a primer for introducing concepts and a resource for further study.
\end{abstract}

Keywords: thulium, holmium, energy transfer, 2 micron lasers. 


\section{Introduction}

An active area of laser research since the 1970's has been in the development of efficient laser sources operating around $2 \mu \mathrm{m}$. Singly doped holmium (Ho) and thulium (Tm), as well as co-doped Tm:Ho systems have been investigated for this purpose. The 2 $\mu \mathrm{m}$ wavelength region possesses many features that make it attractive for laser remote sensing applications, especially in high-energy-per-pulse mode, as well as medical applications. It corresponds to the eye-safe region of the spectrum, and also matches the absorption wavelength of atmospheric constituents such as $\mathrm{CO}_{2}$ and water vapor. The favorable absorption in water also makes such lasers useful for medical applications. A spectrum of the optical absorption in water is shown in Fig. 1, based on measurements by Hale and Querry. [1] As can be seen, there is a strong peak near $2 \mu \mathrm{m}$.

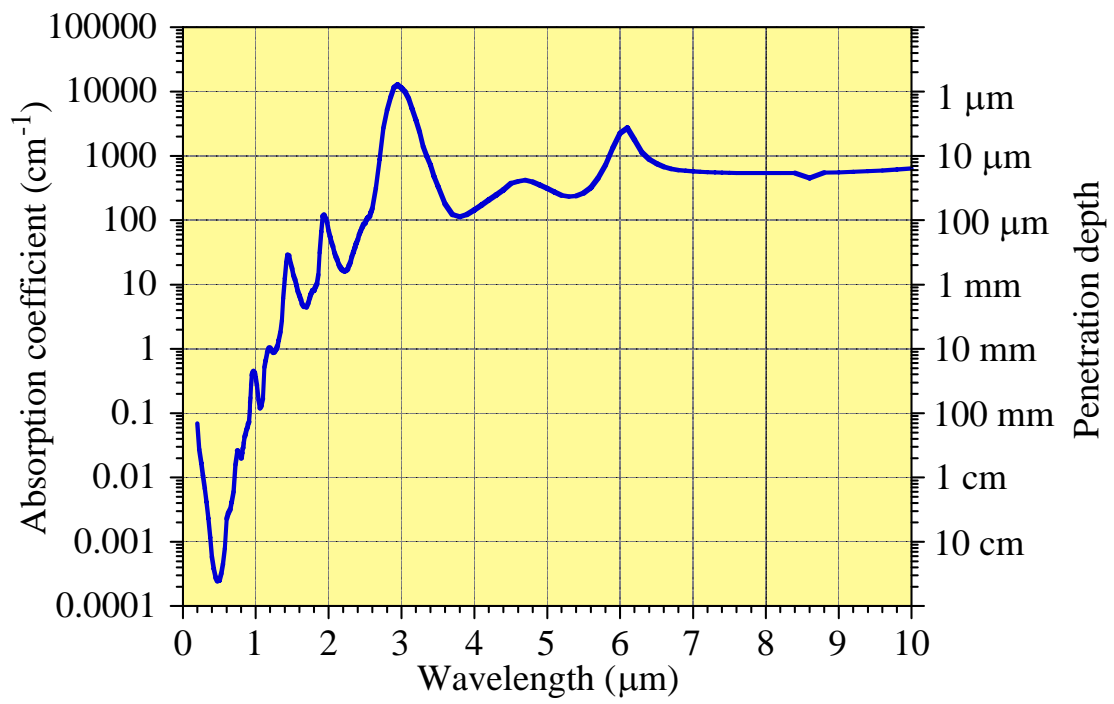

Figure 1. Optical absorption in water (ordinate axis scale is logarithmic).

Tm and Ho lasers operating in the near infrared around $2 \mu \mathrm{m}$ are quasi-four level lasers, and as such, possess a non-negligible population in the lower laser level. As a result, they definitely present a challenge in this regard due to a significant level of pump excitation required to overcome the population in the lower laser level and achieve inversion. In addition, the gain in Tm and Ho lasers is significantly less than the gain in standard Nd lasers operating around $1.06 \mu \mathrm{m}$. These are some of the issues to be faced in the development of efficient $2 \mu \mathrm{m}$ laser sources. Quasi-four level lasers will be discussed in Section 4. 
In Tm:Ho co-doped materials a high efficiency can be achieved using Tm to absorb the laser diode radiation, promoting self-quenching of Tm to produce 2 excited Tm atoms for every absorbed pump photon, and promoting energy transfer to Ho which has a higher gain than Tm for most laser materials. Deleterious processes can also occur, including up-conversion that promotes an excited Ho atom out of the upper laser level. All of these processes are dependent on the choice of the laser material. Consequently, the choice of the laser material is critical. LuAG and LuLF, two materials developed at NASA Langley Research Center, have been shown to offer advantages over YAG and YLF. [2, 3]

This review will cover the fundamental physics on a variety of issues in regards to the spectroscopy of Tm and Ho systems. The laser dynamics will also be covered from the perspective of the theoretical aspects of quasi-four level lasers, energy transfer and modeling. With regards to laser performance, emphasis will be placed on diode pumped Tm:Ho co-doped lasers and Tm lasers, which can be used as a pump source for Ho only lasers. This will provide for a broad based discussion of the interesting complexity of Tm and Ho doped materials, which has continued to be an active area of interest in laser physics research

\section{Applications}

Laser remote sensing can be divided into four categories depending on the type of remote sensor. Simplest is the lidar or light detection and ranging. Lidar operates much similar to radar except that aerosol particles suspended in the air provide the return signal. Lidar can measure the aerosol density, of which a primary constituent is water vapor. Differential absorption lidar, DIAL, uses a two-wavelength lidar transmitter and receiver to measure the density of gaseous atmospheric constituents. One of the wavelengths is tuned to an absorption feature of the atmospheric constituent of interest while the other is tuned to a region where there is no absorption. With a ratio of the returns, the density of the atmospheric constituent can be deduced as a function of range. Wind velocity can be measured using a lidar and heterodyne detection. Aerosol particles travel essentially as fast as the air. When they scatter the incident laser radiation, they impart a slight Doppler shift to the returning radiation. Using heterodyne detection, the Doppler shift can be measured and thus the wind velocity can be inferred. Extremely accurate distance measurements can be made using altimetry techniques. In this case, extremely short laser pulses are used to increase the accuracy of the measurement. By ranging to satellites, tectonic plate motion and the thickness of the ice cap can be measured.

2- $\mu \mathrm{m}$ lasers are the ideally suited for mid infrared remote sensing to monitor the health of Planet Earth and for exploration on other planets. 2- $\mu \mathrm{m}$ lasers can be used 
directly for the remote sensing of wind using heterodyne technology and both water and carbon dioxide using DIAL technology. Wind sensing is very important for weather prediction, storm tracking, airline fuel efficiency and airline safety. Water is important for weather forecasting and climate predictions. Carbon dioxide and water are the primary contributors to the green house effect. Also, 2- $\mu$ m lasers make a nearly ideal pump sources to drive parametric oscillators and amplifiers. With these devices, remote sensing of other important atmospheric species, including methane and carbon monoxide, are possible. In addition, these mid-infrared devices can be used in homeland security for the detection of chemical and biological hazards.

Medical applications of lasers depend on the interaction of light with biological tissue. The effects of a given laser wavelength on the tissue depends on the degree to which it can be absorbed and how rapidly the energy is delivered. The laser itself is only an optical device and contains no inherent heat. When it interacts with tissue, the radiation is absorbed and its energy is converted into motion of the atoms and molecules in the tissue. It's rather like the way a microwave oven works, only with radiation of different frequencies. Mid-infrared lasers have large absorption coefficients in water (see Fig. 1), the major component of biological tissue, and can generate substantial heating in the tissue sufficient enough to break the chemical bonds, making them effective cutting tools and also cauterizing incisions in the process. This makes them ideal for surgical procedures where a lot of bleeding occurs.

$2-\mu \mathrm{m}$ lasers are used in arthroscopy (cartilage repair in joints), urology (prostate ablation, kidney and bladder stones), dentistry and ophthalmology (eye surgery). It has been found to be especially useful for cutting and shaping all types of cartilage in fluidfilled joints using non-invasive arthroscopic techniques. The delivery of the laser by fiber in non-invasive ways is aided by it relatively good transmission in standard silica fibers, where the transmission of begins to tail off beyond $2 \mu \mathrm{m}$.

\section{Historical Development}

The first demonstration of stimulated emission in Tm and Ho ions was performed at Bell Laboratories in 1962. [4, 5] They produced stimulated emission in $\mathrm{Ho:CaWO}{ }_{4}$ on the ${ }^{5} \mathrm{I}_{7} \rightarrow{ }^{5} \mathrm{I}_{8}$ transition at $2.06 \mu \mathrm{m}$ and Tm:CaWO 4 on the ${ }^{3} \mathrm{~F}_{4} \rightarrow{ }^{3} \mathrm{H}_{6}$ transition at 1.91 $\mu \mathrm{m}$. These early systems were lamp pumped and the experiments performed at $77 \mathrm{~K}$. Many of the papers on stimulated emission in the early 1960's refer to these devices as masers. The meaning of this term has changed since its introduction. Today, these devices are called lasers, a terminology coined by Gordon Gould. His thirty-year battle with the United States patent office over the invention of lasers eventually awarded him a patent on optically-pumped laser amplifiers in 1979. This is another story, however, 
detailed in Nick Taylor's book. [6] In 1965, researchers at Bell Labs produced the first Tm:Ho co-doped laser, an $\alpha \beta$-YAG $\left(\mathrm{Y}_{1.25} \mathrm{Er}_{1.5} \mathrm{Tm}_{0.2} \mathrm{Ho}_{0.5} \mathrm{Al}_{5} \mathrm{O}_{12}\right)$ operating at $2.1 \mu \mathrm{m}$. This was a cw tungsten lamp pumped system also operating at 77K. [7]. In 1971 researchers at Sanders Associates produced an $\alpha \beta$-YLF $\left(\mathrm{Y}_{0.416} \mathrm{Er}_{0.5} \mathrm{Tm}_{0.67} \mathrm{Ho}_{0.017} \mathrm{~F}_{4}\right)$ operating at $2.06 \mu \mathrm{m}$. This was a flashlamp-pumped pulsed laser, again operating at $77 \mathrm{~K}$. [8] The first room temperature Tm:Ho laser was demonstrated by researchers at the Universitat Hamburg in 1985. [9] This was a cw Krypton-pumped laser operating at 2.1 $\mu \mathrm{m}$ in Cr:Tm:Ho:YSAG and Cr:Tm:Ho:YSGG. The introduction of diode lasers in the 1980's was a perfect match for Tm:Ho solid-state lasers. The good overlap of diode laser wavelengths with Tm absorption in the ${ }^{3} \mathrm{H}_{4}$ manifold made these ideal excitation sources. In addition, Tm ions have a self-quenching process, the so-called "two-for-one" process where 2 laser ions in the $\mathrm{Tm}^{3} \mathrm{~F}_{4}$ manifold are produced for each pump photon in the ${ }^{3} \mathrm{H}_{4}$ manifold. The first experiments utilizing diode pumping of Tm:Ho materials were done at the Naval Research Laboratory. [10,11] They demonstrated diode-pumped $2 \mu \mathrm{m}$ laser action in Tm:Ho:YAG and Tm:Ho:YLF. From the first demonstration of stimulated emission in Tm and Ho materials in the early 1960's to the subsequent research that followed, eventually led to the first eye-safe coherent lidar in 1991. [12] This lidar system was based on a diode pumped Tm:Ho:YAG at $2.1 \mu \mathrm{m}$. The Tm:Ho technology reached impressive performance levels in 2006 [13] when researchers at NASA Langley Research Center produced 1 Joule per pulse at $2.06 \mu \mathrm{m}$ in Tm:Ho:LuLF, a material invented and developed at NASA Langley. [3]

\section{Quasi-Four Level Lasers}

Tm lasers operating on the ${ }^{3} \mathrm{~F}_{4} \rightarrow{ }^{3} \mathrm{H}_{6}$ transition $(\sim 1.9 \mu \mathrm{m})$ and Ho lasers operating on the ${ }^{5} \mathrm{I}_{7} \rightarrow{ }^{5} \mathrm{I}_{8}$ transition $(\sim 2.0 \mu \mathrm{m})$ are referred to here as quasi-four level lasers. This terminology can be best understood by an analysis of the laser gain. The small signal gain coefficient, $\mathrm{g}_{0}$, is given by [14]

$$
g_{0}=\sigma_{e}\left[\gamma N_{2}-(\gamma-1) C_{A} N_{s}\right]
$$

where $\sigma_{e}$ is the effective stimulated emission cross section, and $\mathrm{N}_{2}$ is the population in the upper laser manifold. $C_{A} N_{s}$ is the product of the concentration of active atoms and the number density of the sites where the active atoms can reside. The term $\gamma=1+f_{l} / f_{\mathrm{u}}$, where $f_{l}$ and $f_{\mathrm{u}}$ are the thermal Boltzmann factors in the lower and upper laser levels, respectively. The factor $\gamma=1$ for a true four level laser and $\gamma=2$ for a true three level laser. Therefore, a quasi-four level laser has a value of $\gamma$ that is closer to 1.0 than 2.0. To see why this is so, consider Fig. 2. This figure shows the energy level schematic for three 
and four level lasers. An example of a three level laser is the $\mathrm{Cr}: \mathrm{Al}_{2} \mathrm{O}_{3}$ (ruby) laser operating on the ${ }^{2} \mathrm{E} \rightarrow{ }^{4} \mathrm{~A}_{2}$ transition at $0.69 \mu \mathrm{m}$. An example of a four level laser is the $\mathrm{Nd}$ :YAG laser operating on the ${ }^{4} \mathrm{~F}_{3 / 2} \rightarrow{ }^{4} \mathrm{I}_{11 / 2}$ transition at $1.064 \mu \mathrm{m}$. As can be seen from this figure, the lower laser level in a three level laser is the ground state and, as such, has some thermal population. In a four level laser the lower laser level is an excited state with no thermal population. Since $\gamma=1+f_{l} / f_{\mathrm{u}}$ then $\gamma$ must be 1 when $f_{l}=0$ for a four level laser and 2 when $f_{l}=f_{\mathrm{u}}$ for a three level laser. Although a quasi-four level laser resembles the three level laser in structure, it is actually quasi-four level because $f_{l}$ is close to small, but not negligible. In other words, it looks like a three level laser but behaves more nearly like a four level laser, hence the name quasi-four level. The criteria for the terminology is that for $\gamma<1.5$, the laser is quasi-four level and for $\gamma>1.5$ it is quasi-three level.

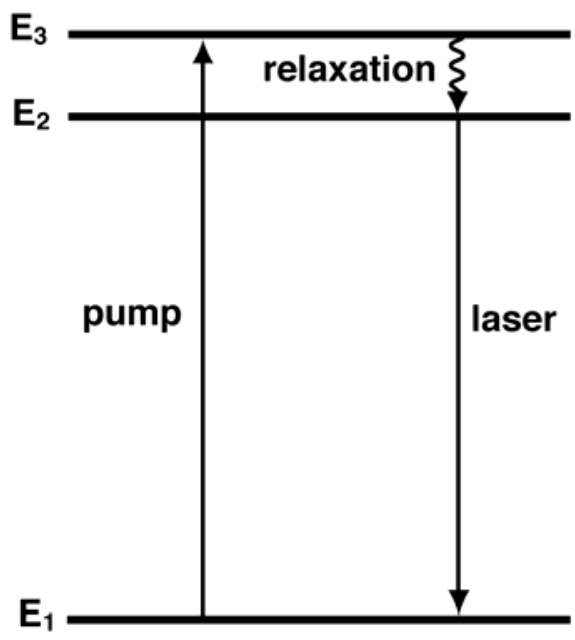

(a) Three level laser

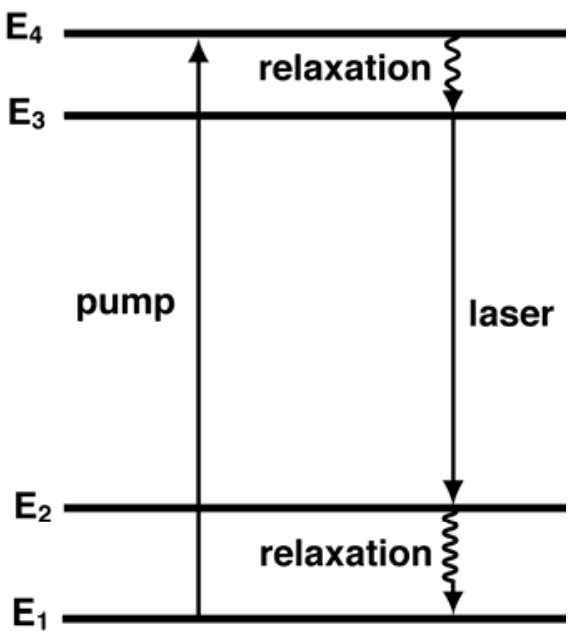

(b) Four level laser

Figure 2. Schematic of (a) three level and (b) four level lasers.

Some examples of quasi-four level lasers besides the Tm: ${ }^{3} \mathrm{~F}_{4} \rightarrow{ }^{3} \mathrm{H}_{6}(\sim 1.9 \mu \mathrm{m})$ and Ho: ${ }^{5} \mathrm{I}_{7} \rightarrow{ }^{5} \mathrm{I}_{8}(\sim 2.0 \mu \mathrm{m})$ include Nd ${ }^{4} \mathrm{~F}_{3 / 2} \rightarrow{ }^{4} \mathrm{I}_{9 / 2}(\sim 0.94 \mu \mathrm{m}), \mathrm{Yb}:{ }^{2} \mathrm{~F}_{5 / 2} \rightarrow{ }^{2} \mathrm{~F}_{7 / 2}(\sim$ $1.0 \mu \mathrm{m})$, and Er: ${ }^{4} \mathrm{I}_{13 / 2} \rightarrow{ }^{4} \mathrm{I}_{15 / 2}(\sim 1.5 \mu \mathrm{m})$. These types of lasers present manageable challenges due to the small thermal occupation factors residing in the lower laser level. The emission of phonons can add to the heat load, however, which adversely affects quasi-four-level lasers by increasing the lower laser level population and, therefore, increasing the threshold of the laser. The effect of the phonon energy regarding energy transfer processes is an important consideration as well. This is true for Tm lasers and the two-for-one self-quenching process mentioned in the last section. Energy transfer processes in Tm and Ho singly and co-doped systems are covered in the next section. 


\section{Energy Transfer}

When a solid-state material containing optically active ions is exposed to a radiation source, electronic states can be excited if the ions absorb some of the photons in the radiation field. At some later time the electronic states de-excite through the emission of photons, which show up as light, or they can give up phonons to the crystal lattice, which show up as heat. This simple picture works well if the ions can be considered as isolated systems, which would be the case for very small ion concentrations and the ions are well separated from each other. As the concentration of dopant ions is increased, however, the spacing between ions becomes small enough to allow the ions to interact with each other. It is this interaction between ions that allows for the transfer of energy from one ion to another.

Energy transfer can result from various processes. Those that are of interest for solid-state laser materials include radiative and nonradiative processes. Radiative energy transfer involves the emission of a photon by one ion (the donor or sensitizer) and subsequent absorption of that photon by another ion (the acceptor or activator). This type of behavior is well understood in terms of the usual emission and absorption processes. However, the migration of radiative energy does produce observable effects such as radiative trapping, which can lead to lengthening of the observed fluorescent lifetime. However, since this process does not involve any coupling between the two ions they don't affect the total emission probability of one another. The acceptor ion simply absorbs the photon emitted by the donor ion. Nonradiative energy transfer is much more interesting from a physics point of view. In this process the excitation energy from one ion is transferred to another ion nonradiatively, essentially stealing part or all of its energy. This can occur through a Coulomb interaction via the electromagnetic field, or an exchange interaction via the overlap of the electron charge clouds. The former can be described quantum mechanically as exchange of a virtual photon since the transitions occur simultaneously for the two ions. It is an 'action at a distance' mechanism and can be interpreted classically, such that classical analogies are possible, as the long range interaction of oscillating dipoles. Förster [15] first described this in terms of a dipoledipole interaction. Dexter [16] later extended this to include multipole interactions and the exchange interaction. These energy transfer processes for ions in solids are sometimes referred to as "Förster-Dexter theory".

Non-radiative energy transfer can be a resonant one if the energy levels of the absorbing ions (donor) overlap the energy levels where emission occurs in the acceptors. The transfer can also be non-resonant if the phonons in the lattice are of sufficient energy to assist the transfer of energy by the creation or annihilation of phonons from the lattice such that the energy mismatch is compensated. Energy conservation is maintained in this 
way. Through phonon assistance, many energy transfer processes can be realized, even when the transitions between the donor and acceptor are not very resonant. Consideration of energy transfer processes involve Tm-Tm transfer, Ho-Ho transfer and Tm-Ho transfer. These are considered in the following sub-sections.

\subsection{Ho-Ho energy transfer}

Ho:Ho energy transfer processes can occur by which two Ho atoms in the ${ }^{5} \mathrm{I}_{7}$ manifold interact to promote one of the atoms to the ${ }^{5} \mathrm{I}_{5}$ manifold and to demote the other Ho atom to the ${ }^{5} \mathrm{I}_{8}$ manifold. This is an upconversion process denoted by $\mathrm{p}_{77}$ in Fig. 3. Conversely, a Ho atom in the ${ }^{5} \mathrm{I}_{5}$ manifold, and a Ho atom in the ${ }^{5} \mathrm{I}_{8}$ manifold can interact to generate two Ho atoms in the ${ }^{5} \mathrm{I}_{7}$ manifold. This is the reverse of $\mathrm{p}_{77}$ upconversion, and is a self-quenching process denoted by $\mathrm{p}_{58}$. All energy transfer processes have a reverse process, but their effectiveness depends on the particular energy level structure of the ion in a given host material. Ho:Ho upconversion rates, $\mathrm{p}_{77}$, have been measured in Ho:YAG, Ho:LuAG and Ho:YLF. [17]

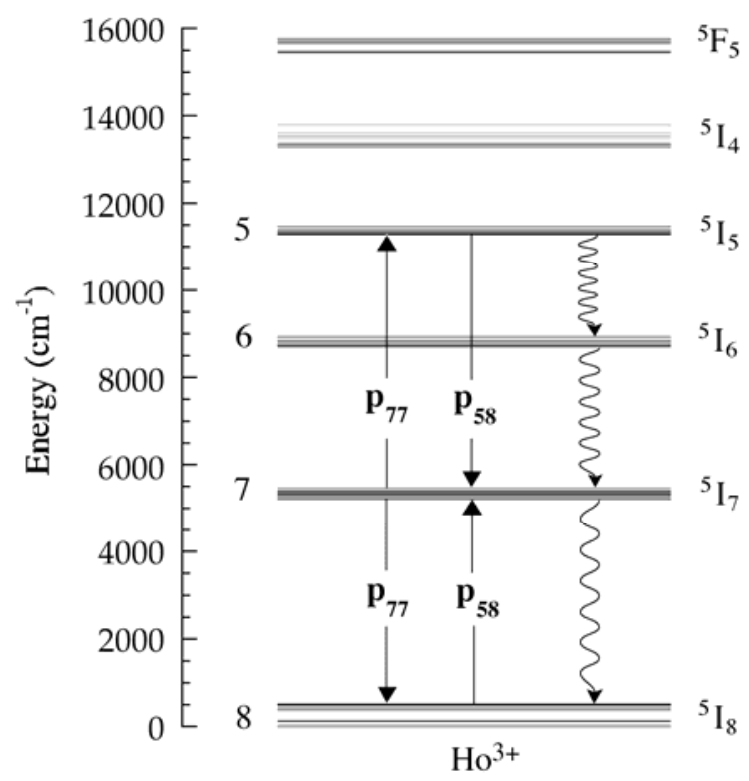

Figure 3. Energy level schematic of Ho-Ho energy transfer processes.

Because the ${ }^{5} \mathrm{I}_{5}$ manifold quickly decays to the ${ }^{5} \mathrm{I}_{6}$ manifold, usually by nonradiative transitions, in this case the $\mathrm{p}_{77}$ process has a low probability of being reversed. As a consequence, this Ho:Ho upconversion is a deleterious process. Because the upconversion process involves two closely spaced Ho atoms in the ${ }^{5} \mathrm{I}_{7}$ manifold, the effect is most likely to be observed when the population density of the ${ }^{5} \mathrm{I}_{7}$ manifold is high. Thus upconversion is most important when the energy storage is high, a situation 
common for Q-switching. Ho:Ho upconversion sets a limit on the energy storage of Hoonly lasers and thus limits both the Q-switched energy and the laser efficiency.

\subsection{Tm-Tm energy transfer}

The decay dynamics of Tm ions from the ${ }^{3} \mathrm{H}_{4}$ manifold exhibit non-exponential decay behavior that strongly shortens with increasing Tm concentration. This is a result of a self-quenching process, also referred to as cross-relaxation [18], denoted by the process $\mathrm{p}_{41}$ in Fig. 4. In this process an excited ion in the ${ }^{3} \mathrm{H}_{4}$ manifold interacts with a nearby ground state ion in the ${ }^{3} \mathrm{H}_{6}$ manifold and undergoes a transfer of energy, resulting in two ions in the ${ }^{3} \mathrm{~F}_{4}$ manifold. This cross-relaxation mechanism, interpreted as the energy transfer $\operatorname{Tm}\left({ }^{3} \mathrm{H}_{4} \rightarrow{ }^{3} \mathrm{~F}_{4}\right) ; \operatorname{Tm}\left({ }^{3} \mathrm{H}_{6} \rightarrow{ }^{3} \mathrm{~F}_{4}\right)$, is a phonon assisted transfer in this case since the energy levels exhibit no resonance overlap. The reverse process is shown as $\mathrm{p}_{22}$. Tm:Tm cross relaxation rates, $\mathrm{p}_{41}$, are measured in Tm:YAG [19] and Tm:YLF [20].

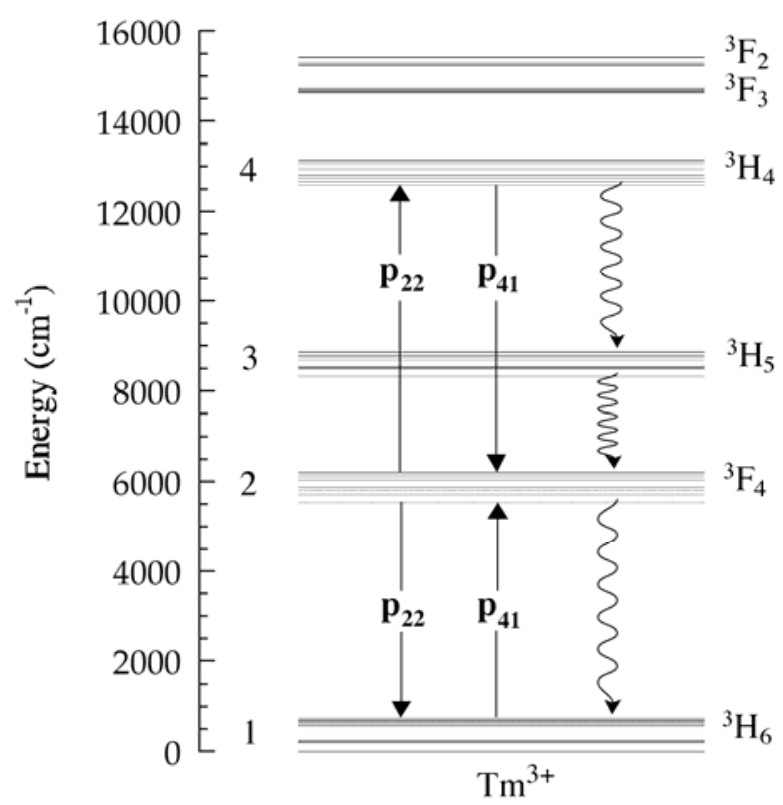

Figure 4. Energy level schematic of Tm-Tm energy transfer processes.

In Tm doped laser materials non-radiative decay can compete with selfquenching, however, limiting its so-called 'two-for-one' quantum efficiency. Lower phonon materials, therefore, are more advantageous. In addition, Tm concentration also plays a role. If the concentration is kept low, self-quenching is inhibited. While this is not advantageous for lasers operating on the ${ }^{3} \mathrm{~F}_{4} \rightarrow{ }^{3} \mathrm{H}_{6}$ transition around $1.9 \mu \mathrm{m}$, it is advantageous for lasers operating on the ${ }^{3} \mathrm{H}_{4} \rightarrow{ }^{3} \mathrm{~F}_{4}$ transition around $1.5 \mu \mathrm{m}$.

The approach for Tm lasers excites the ${ }^{3} \mathrm{H}_{4}$ manifold around $0.79 \mu \mathrm{m}$, where 
diode lasers are commercially available. If each pump photon only produced one Tm atom in the upper laser manifold, the ${ }^{3} F_{4}$, then the ratio of pump to laser photon energies would limit the efficiency to $\sim 0.4$. However, Tm atoms undergo self-quenching. This produces two Tm atoms in the upper laser manifold. In this situation, the quantum efficiency is ideally 2.0 and the laser efficiency limit increases to $\sim 0.8$, a highly advantageous situation for $1.9 \mu \mathrm{m}$ lasers. Nevertheless, Tm materials generally have relatively low gain as well and better suited for fiber laser applications, which lave long lengths, despite the low gain.

\subsection{Tm-Ho energy transfer}

The situation becomes much more complex in Tm:Ho co-doped systems. The task of identifying all channels of energy transfer processes is a formidable one that has yet to be entirely solved. Ideally, the best method of approach would be to excite many different manifolds and observe the luminescence and decay characteristics of the various other manifolds under different pumping conditions and over a range of temperatures. Such experiments ideally would be done with a suitable range of dopant ion concentrations as well. This is a rather tall order, so to speak, and in practice is not always possible due to constraints on the availability of the necessary experimental apparatus and samples. Nevertheless, a large number of studies, each concentrating on some specific aspect of Tm:Ho systems has produced some coherent picture of the dynamics. Fig. 5 illustrates the complexity of energy transfer in Tm:Ho materials and displays some of the more important processes affecting Tm:Ho lasers.
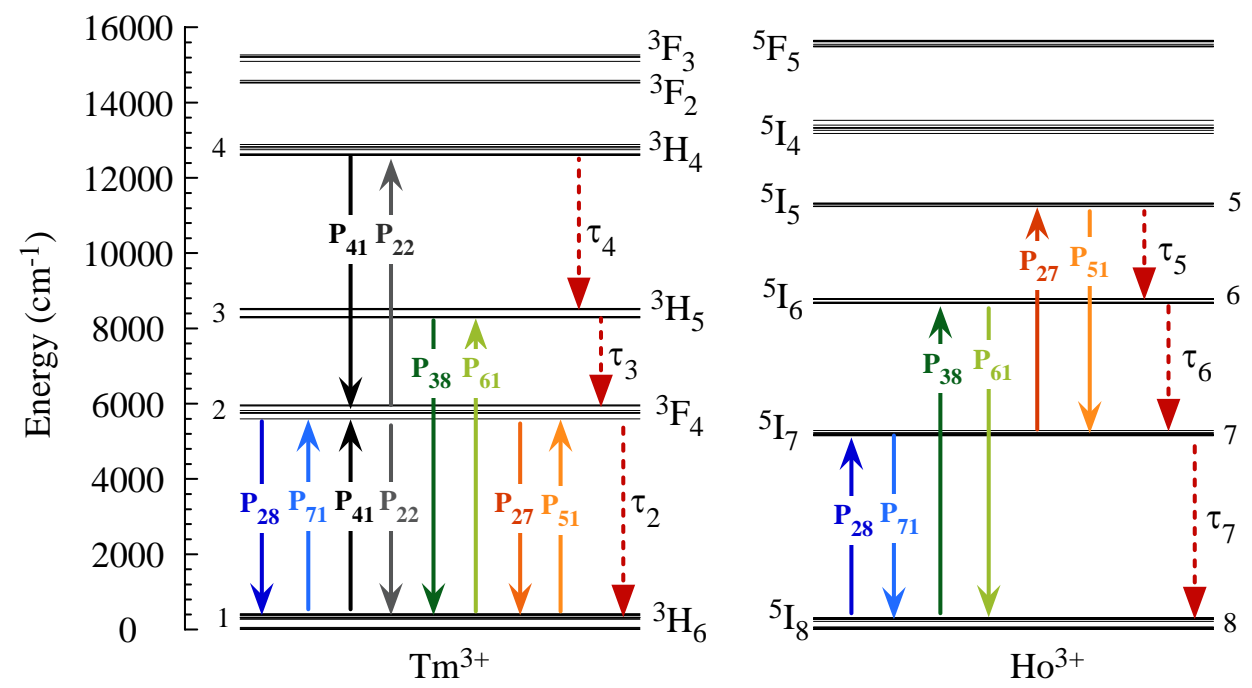

Figure 5. Energy level schematic of Tm-Ho energy transfer processes. 
The main channel of energy transfer between Tm and Ho is the resonant transfer from the $\mathrm{Tm}^{3} \mathrm{~F}_{4}$ to the $\mathrm{Ho}^{5} \mathrm{I}_{7}$, denoted by the process $\mathrm{p}_{28}$. The reverse of this process is $\mathrm{p}_{71}$, the backtransfer from the $\mathrm{Ho}^{5} \mathrm{I}_{7}$ to the $\mathrm{Tm}^{3} \mathrm{~F}_{4}$. These energy transfer processes are illustrated in Fig. 6. At early times after excitation of the $\mathrm{Tm}^{3} \mathrm{~F}_{4}$ manifold, a sharp rise in $\mathrm{Ho}^{5} \mathrm{I}_{7}$ population as accompanied by a sharp decline in $\mathrm{Tm}^{3} \mathrm{~F}_{4}$ population. At long times after the pulse, the populations are thermalized and decay at the same rate. [21, 22]
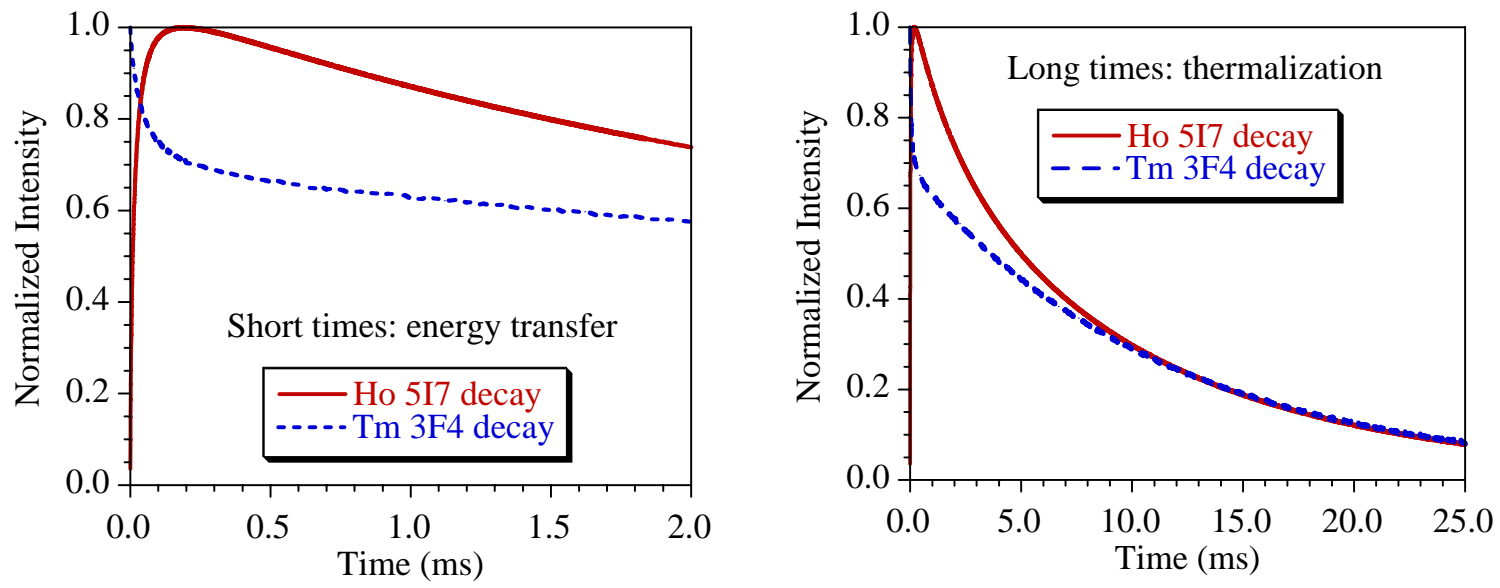

Figure 6. Tm:Ho decay dynamics at short and long times after excitation of $\mathrm{Tm}^{3} \mathrm{~F}_{4}$.

The situation can be explained in the following way. After a pulse, the Tm and Ho ions have a certain amount of energy as a combined system and an agreement through Boltzmann statistics to maintain the distribution between the two. Even though the total amount of energy in the $\mathrm{Tm}{ }^{3} \mathrm{~F}_{4}$ and $\mathrm{Ho}^{5} \mathrm{I}_{7}$ manifolds is decreasing, the distribution between the two eventually reaches quasi-thermal equilibrium and the Tm and Ho ions decay at the same rate. By looking at the early parts of the decay curve, we can catch Tm and Ho in the act of sharing their energy. At later times they are still sharing energy, but have essentially become thermalized and each displays the same rate of decay.

While Tm:Ho laser materials represent a storied tale full of interesting complexity and continue to be of scientific interest regarding energy transfer processes, the mainstay of laser operation on the $\mathrm{Ho}^{5} \mathrm{I}_{7} \rightarrow{ }^{5} \mathrm{I}_{8}$ transition at $\sim 2 \mu \mathrm{m}$ relies on two separate energy transfer processes. Excitation of the $\mathrm{Tm}^{3} \mathrm{H}_{4}$ is followed by cross relaxation, producing two excitations in the $\mathrm{Tm}{ }^{3} \mathrm{~F}_{4}$ for each pump photon. This is followed by direct energy transfer from the $\mathrm{Tm}^{3} \mathrm{~F}_{4}$ to $\mathrm{Ho}^{5} \mathrm{I}_{7}$. It is a fairly efficient laser way of doing things, but there may be some advantages to using a Tm laser to pump Ho directly, rather than rely on the Tm $\leftrightarrow$ Ho energy sharing in co-doped Tm:Ho materials. This subject has been of interest in recent years and will be covered in Section 7. 


\section{Laser Modeling}

The modeling of a laser requires many input parameters to account for the variety of processes that contribute to the physics of achieving a population inversion. A fairly comprehensive set of parameters is usually needed to describe any laser system. For Tm:Ho systems, this can be a challenge with regards to the energy transfer processes. In order to assess the relative importance of parameters used, a laser model is constructed as an adaptive tool to assess laser performance. It is not something born all at once, and is developed over time, using spectroscopic parameters of experimental origin and those derived from theory, as well as laser performance observations as well.

The modeling of Tm:Ho laser systems has been the subject of a number of articles in the past. [23-32] The approach is usually to construct a set of rate equations governing the populations of all the manifolds that have an impact on the laser performance. In the case of Tm:Ho co-doped systems, the number of manifolds to consider can number 10 or more. This requires knowledge of a great many energy transfer and decay parameters. Inclusion of all these parameters in laser models becomes problematic not because the equations are cumbersome. This is not a problem for modern computers. The main difficulty is in accurately measuring or predicting many of these parameters. The approach is then to make some assumptions regarding the dynamics of interaction of the Tm and Ho ions. The model, once constructed, can then be used as a tool towards new interpretation of the dynamics in the Tm:Ho laser systems. In an adaptive approach, modeling will predict laser performance and aid in assessment of input parameters, refining both the understanding of the fundamental processes and the laser predictions.

Rather than reiterate laser models here, it is more instructive to briefly describe the construction of rate equation models in the context of quasi-four systems. Rate equations appropriate for quasi-four level lasers in general can be written as [33]

$$
\begin{aligned}
& \frac{d N_{2}}{d \mathrm{t}}=-\frac{N_{2}}{\tau_{2}}+R_{2}-\sigma \frac{c}{n}\left(f_{2} N_{2}-f_{1} N_{1}\right) \phi \\
& \frac{d N_{1}}{d \mathrm{t}}=\frac{N_{2}}{\tau_{2}}-R_{2}+\sigma \frac{c}{n}\left(f_{2} N_{2}-f_{1} N_{1}\right) \phi \\
& \frac{d \phi}{d \mathrm{t}}=-\frac{\phi}{\tau_{c}}+\sigma \frac{c}{n} \frac{l}{[(n-1) l+L]}\left(f_{2} N_{2}-f_{1} N_{1}\right) \phi
\end{aligned}
$$

where $\mathrm{N}_{2}$ and $\mathrm{N}_{1}$ are the population densities of the upper and lower laser manifolds, respectively, and $\phi$ is the photon density. $\tau_{2}$ is the lifetime of the upper laser level, $R_{2}$ is the pumping rate, $f_{1}$ and $f_{2}$ are the Boltzmann fractions of the lower and upper laser 
levels, respectively, $\sigma$ is the emission cross section of the laser transition, $\mathrm{c}$ is the speed of light, $\mathrm{n}$ is the index of refraction, $l$ is the length of the gain medium and $\mathrm{L}$ is the length of the resonator. The inverse of the cavity lifetime, $\tau_{c}$, is given by

$$
\frac{1}{\tau_{c}}=\frac{C}{2 L} \ln \left(R_{m} R_{L}\right)
$$

where $\mathrm{R}_{\mathrm{m}}$ represents the output mirror loss and $\mathrm{R}_{\mathrm{L}}$ represents losses in the laser resonator. Equations (2) to (4) represent the fundamental physics governing quasi-four level lasers.

If a majority of the optically active atoms in the system reside in the upper or lower laser manifold then $N_{1}+N_{2} \cong \mathrm{C}_{\mathrm{n}} \mathrm{N}_{\mathrm{s}}$, where $\mathrm{C}_{\mathrm{n}}$ is the dopant concentration of laser ions and $\mathrm{N}_{\mathrm{s}}$ is the density of sites available for doping. So, substituting $N_{1}=\mathrm{C}_{\mathrm{n}} \mathrm{N}_{\mathrm{s}}-N_{2}$, using $\gamma=1+f_{1} / f_{2}$, and $\sigma_{\mathrm{e}}=f_{2} \sigma$, gives

$$
\begin{aligned}
& \frac{d N_{2}}{d \mathrm{t}}=-\frac{N_{2}}{\tau_{2}}+R_{2}-\sigma_{e} \frac{C}{n}\left[\gamma N_{2}-(\gamma-1) C_{n} N_{s}\right] \phi \\
& \frac{d \phi}{d \mathrm{t}}=-\frac{\phi}{\tau_{\mathrm{c}}}+\sigma_{e} \frac{c}{n} \frac{l}{[(n-1) l+L]}\left[\gamma N_{2}-(\gamma-1) C_{n} N_{s}\right] \phi
\end{aligned}
$$

This set of coupled equations can be solved to find the population density $\mathrm{N}_{2}$ and closed form solutions for the threshold and slope efficiency in normal mode and Qswitched operation. [33] A more versatile description of the laser dynamics that can produce simulations of laser performance for more complicated systems require the inclusion of energy transfer and decay processes into the laser rate equations. The rate equations can be formulated by remembering that whenever one manifold loses atoms, another must gain the same number. Taking Tm as a simple example and referring to Fig. 3 , the rate equations describing these energy transfer processes can be constructed as follows

$$
\begin{aligned}
& \frac{d N_{4}}{d \mathrm{t}}=-\frac{N_{4}}{\tau_{4}}-p_{41} N_{4} N_{1}+p_{22} N_{2}^{2} \\
& \frac{d N_{3}}{d t}=-\frac{N_{3}}{\tau_{3}}+\frac{\beta_{43} N_{4}}{\tau_{4}} \\
& \frac{d N_{2}}{d \mathrm{t}}=-\frac{N_{2}}{\tau_{2}}+\frac{\beta_{32} N_{3}}{\tau_{3}}+\frac{\beta_{42} N_{4}}{\tau_{4}}+2 p_{41} N_{4} N_{1}-2 p_{22} N_{2}^{2} \\
& \frac{d N_{1}}{d \mathrm{t}}=\frac{N_{2}}{\tau_{2}}+\frac{\beta_{31} N_{3}}{\tau_{3}}+\frac{\beta_{41} N_{4}}{\tau_{4}}-p_{41} N_{4} N_{1}+p_{22} N_{2}^{2}
\end{aligned}
$$


The $\mathrm{p}_{41}$ self-quenching and reverse $\mathrm{p}_{22}$ processes have been discussed in section 5.2. The numbering convention of the manifolds is the same as in Fig. 3 . These equations can be used to construct a Tm laser model. Alternatively, a Tm:Ho laser model can be constructed. As a simple example, consider direct pumping into level 2, the $\mathrm{Tm}{ }^{3} \mathrm{~F}_{4}$ manifold. Direct energy transfer from Tm to Ho will take place via the process $\mathrm{p}_{28}$ as pictured in Fig. 5. If it is assumed that all Tm and Ho atoms reside in the lowest two manifolds of each ion, then $N_{1}+N_{2}=\mathrm{C}_{\mathrm{Tm}} \mathrm{N}_{\mathrm{s}}$ and $N_{7}+N_{8}=\mathrm{C}_{\mathrm{Ho}} \mathrm{N}_{\mathrm{s}}$. Only the process $\mathrm{P}_{28}$ and its reverse $\mathrm{p}_{71}$ need be considered for this simplified model. The laser rate equations with energy transfer dynamics included are written as

$$
\begin{aligned}
& \frac{d N_{1}}{d \mathrm{t}}=-R_{2}+\frac{N_{2}}{\tau_{2}}+p_{28} N_{2} N_{8}-p_{71} N_{7} N_{1} \\
& \frac{d N_{2}}{d \mathrm{t}}=R_{2}-\frac{N_{2}}{\tau_{2}}-p_{28} N_{2} N_{8}+p_{71} N_{7} N_{1} \\
& \frac{d N_{7}}{d \mathrm{t}}=-\frac{N_{7}}{\tau_{7}}+p_{28} N_{2} N_{8}-p_{71} N_{7} N_{1}-\sigma \frac{c}{n}\left(f_{7} N_{7}-f_{8} N_{8}\right) \phi \\
& \frac{d N_{8}}{d \mathrm{t}}=\frac{N_{2}}{\tau_{2}}-p_{28} N_{2} N_{8}+p_{71} N_{7} N_{1}+\sigma \frac{c}{n}\left(f_{7} N_{7}-f_{8} N_{8}\right) \phi \\
& \frac{d \phi}{d \mathrm{t}}=-\frac{\phi}{\tau_{\mathrm{c}}}+\frac{c}{n} \frac{l}{[(n-1) l+L]} \sigma\left(f_{7} N_{7}-f_{8} N_{8}\right)+b_{s p} \frac{N_{7}}{\tau_{7}}
\end{aligned}
$$

This set of equations cannot be solved in closed form, but are straightforward to solve using numerical techniques and especially well suited to computer simulations. The last term in Eq. 16, $b_{\mathrm{sp}}$, accounts for the spontaneous emission necessary to drive the laser toward stimulated emission and subsequent amplification. It essentially provides a few spontaneously emitted photons to begin the laser process. The more complicated case of pumping into level 4, the $\mathrm{Tm}^{3} \mathrm{H}_{4}$, has been covered previously. [30] The laser model in this case is much more extensive and includes all the processes pictured in Fig. 5. It is seen that the equations are not too difficult to write down, once the processes have been defined. As pointed out earlier, acquiring the necessary parameters can be problematic, requiring measurement and theory for a complete set.

\section{Tm-Pumped Ho Lasers}

Conventionally, flashlamps and laser diode arrays have been used to pump Tm:Ho laser materials. Diode lasers are more commonly used today. Commercially available laser 
diode arrays pump the $\mathrm{Tm}{ }^{3} \mathrm{H}_{4}$ atoms directly that cross relax to the ${ }^{3} \mathrm{~F}_{4}$ manifold, which in turn transfers the energy to the $\mathrm{Ho}^{5} \mathrm{I}_{7}$ atoms that store the energy. Tm, rather than Ho, is pumped because laser diodes operating at wavelengths corresponding to the absorption bands of Tm are commercially available. In essence, Tm:Ho laser materials integrate the highly divergent power produced by the laser diodes over the relatively long pump pulse and can produce a single, short, nearly diffraction limited laser pulse. While this approach yields reasonable efficiencies, the basic physics tend to limit the maximum efficiency.

The reason is that Tm:Ho lasers depend on a variety of energy transfer processes, but not all of them act in beneficial ways. Upconversion can be deleterious to efficiency and shorten the upper laser level lifetime and thus the useful pump time interval, limiting the effective storage time. The $\mathrm{p}_{27}$ process in Fig. 5 is an example of a deleterious upconversion process, especially in Q-switched operation. Also, while Tm $\rightarrow$ Ho energy transfer, $\mathrm{p}_{28}$, provides a channel for transfer of absorbed pump energy in Tm atoms to Ho atoms, the reverse process, $\mathrm{p}_{71}$, remains active for long times after the pump pulse as was illustrated in Fig. 6. This sharing of the absorbed energy by the Ho and Tm atoms limits efficiency because only energy stored in the Ho can be extracted in a single, short pulse. Nominally, only half the energy is available in Ho at the time of Q-switching. Due to the quasi-four level nature of these lasers, the high pump fluences required are limited by upconversion, Tm $\leftrightarrow$ Ho energy sharing and the divergent nature of pump diodes for coupling energy into these materials. In addition, the quantum defect, that is, the ratio of pump wavelength to laser wavelength, in diode laser pumped Tm:Ho lasers is $<0.5$ further limiting their efficiency due to the heat load that results from this. This is especially bad for quasi-four level lasers due to the non-negligible thermal population in the lower laser level.

An attractive alternative, which has been explored in recent years, is to separate the $\mathrm{Tm}$ and Ho atoms in different materials. Tm-pumped Ho lasers are appealing for many reasons. By separating the Ho and Tm, deleterious energy transfers are minimized. This allows an increase in the upper laser lifetime of Ho and more efficient temporal integration in pumping. Also, because the Ho and Tm are separated, all of the pump energy will be stored in the Ho providing much more of the pump energy to be extracted in a single, short pulse. In addition, the Tm laser wavelength is only slightly shorter than the Ho laser wavelength, so the quantum defect is $\sim 1.0$, and less heat is deposited, improving beam quality. This also benefits the quasi-four level nature of the laser and offers an advantage for keeping lower laser level populations at a minimum.

There are a many alternative approaches to consider. One is to use a Tm laser such as Tm:YLF to pump Ho. Another is to use a Tm:fiber laser to pump Ho. These Tm materials are chosen for their good overlap with Ho:YAG or Ho:YLF absorption features. Fiber lasers offer effective spatial integration of the laser diode pump by using a dual clad fiber. Specifically, pump power is confined by a large outer cladding, simplifying the coupling of 
the laser diode arrays to the fiber, while the Tm is confined to a much smaller inner cladding, producing a good beam with which to pump the subsequent Ho laser. Output of the Tm fiber is concentrated onto a Ho laser material to achieve very high pump intensities. Choosing Ho:YAG over Ho:YLF as a laser material has advantages due to its larger Stark level splitting and, hence smaller thermal populations in the lower laser level. As the quantum defect is low, heat deposition is less of a problem and thermal lensing effects associated with YAG are of less concern than in Tm:Ho materials. Some results on these two alternative approaches are presented in the following subsections.

\subsection{Tm:YLF pumping Ho:YAG}

A diode pumped Tm:YLF disc laser end-pumps a Ho:YAG laser rod. The apparatus for this configuration is shown in Fig. 7.

Diode Laser pumped Tm:YLF laser

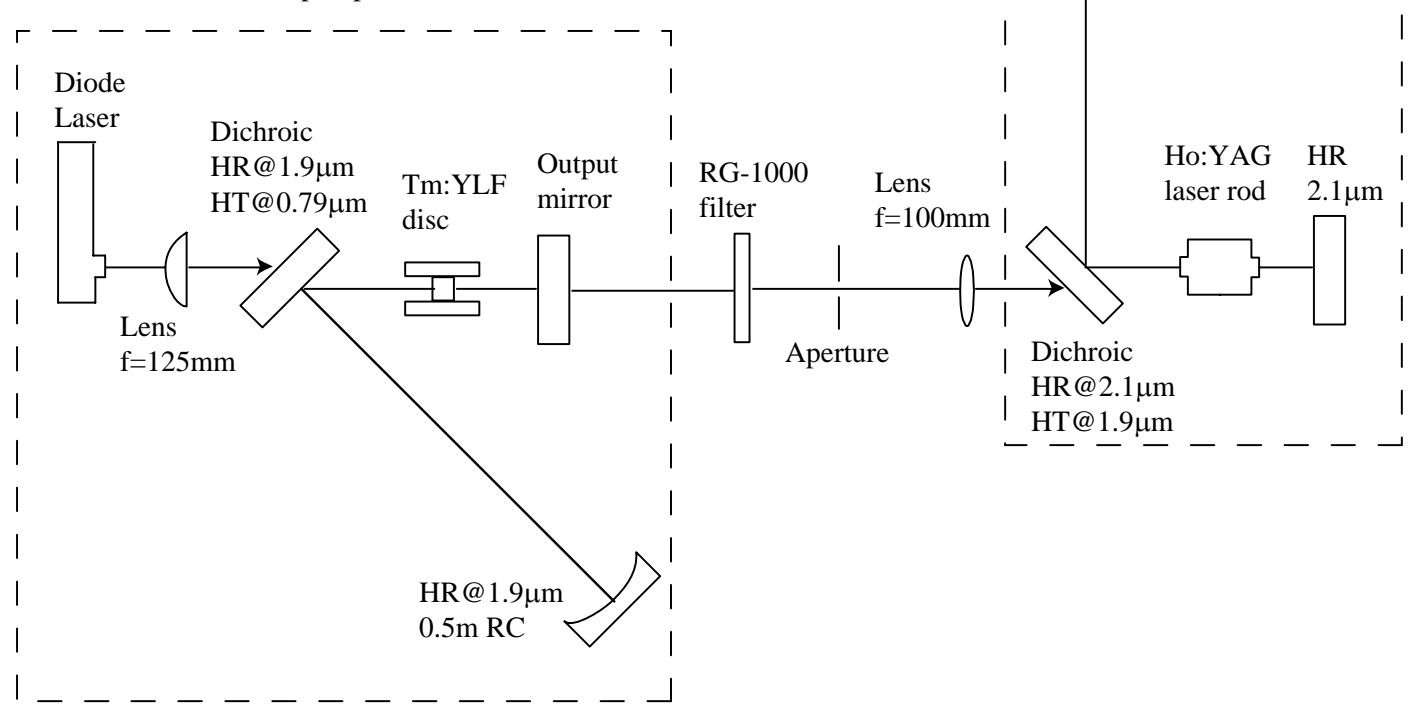

Figure 7. Schematic of a Tm:YLF pumped Ho:YAG Laser.

Pulsed laser measurements were performed on slope efficiency versus Ho rod length, mirror reflectivity and Tm pump wavelength. These are shown in Fig. 8a to 8c. The Ho laser energy versus Tm pump energy for best performance is shown in Fig. 8d. A maximum slope efficiency of $41 \%$ was obtained with a threshold of $3.28 \mathrm{~mJ}$. 

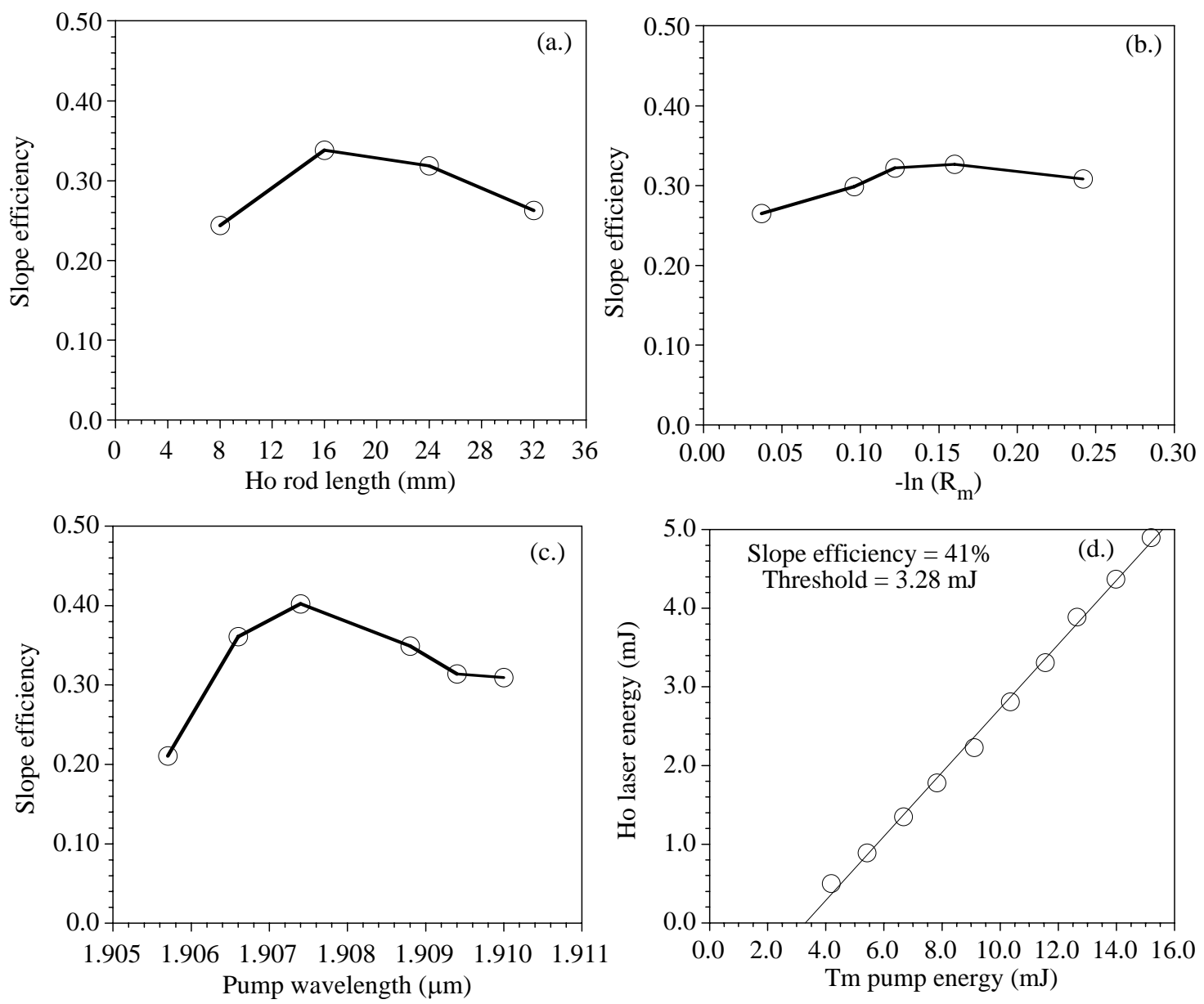

Figure 8. Performance of Tm:YLF pumped Ho:YAG versus Ho rod length (a), mirror reflectivity (b), pump wavelength (c), and pump energy (d).

The best performance occurs for a pump wavelength $\sim 1.907 \mu \mathrm{m}$, corresponding to a peak absorption feature in Ho:YAG. Optimal Ho rod length is $~ 16$ to $20 \mathrm{~mm}$, and optimal output mirror reflectivity is $\sim 0.85$. A nominal concentration of $1.0 \%$ Ho was present in the samples used for these experiments. Lower Ho concentrations, $0.5 \%$ Ho, required longer rods for efficient pump absorption that affected alignment tolerances. This produced lower performances than the $1.0 \%$ Ho laser rods. Modeling of this laser has produced predictions for threshold and slope efficiency that are in reasonable agreement with the experimental results. [34]

\subsection{Tm:fiber pumping Ho:YAG}

A diode laser pumped Tm:fiber laser end-pumps a Ho:YAG laser rod. The apparatus for this configuration is shown in Fig. 9. 


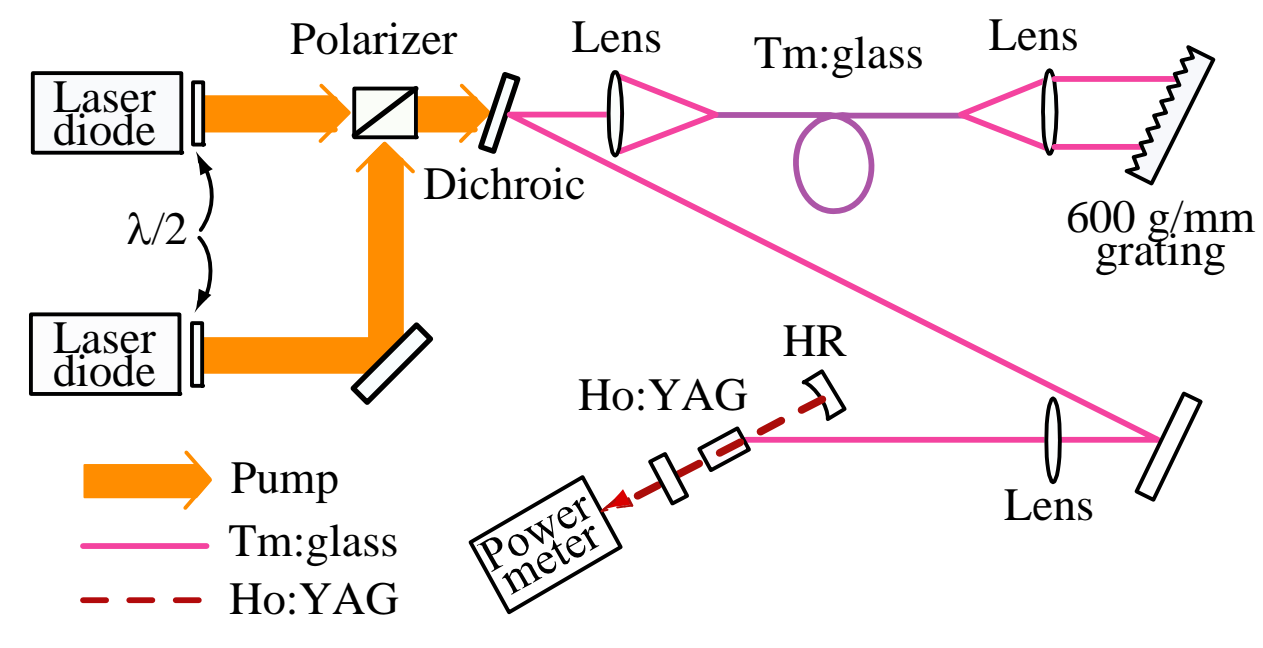

Figure 9. Schematic of a Tm:fiber pumped Ho:YAG Laser.

$\mathrm{Cw}$ laser measurements were performed on the fraction of pump power absorbed in Ho versus Tm pump power and Ho laser power versus Tm:fiber pump power. The results are shown in figures 10a and 10b. A maximum slope efficiency of $37 \%$ was obtained with a threshold of $1.45 \mathrm{~W}$. The product of the absorption efficiency, $\sim 0.40$, and the quantum defect, 0.91 , is calculated to be $\sim 0.36$, close to the observed slope efficiency.
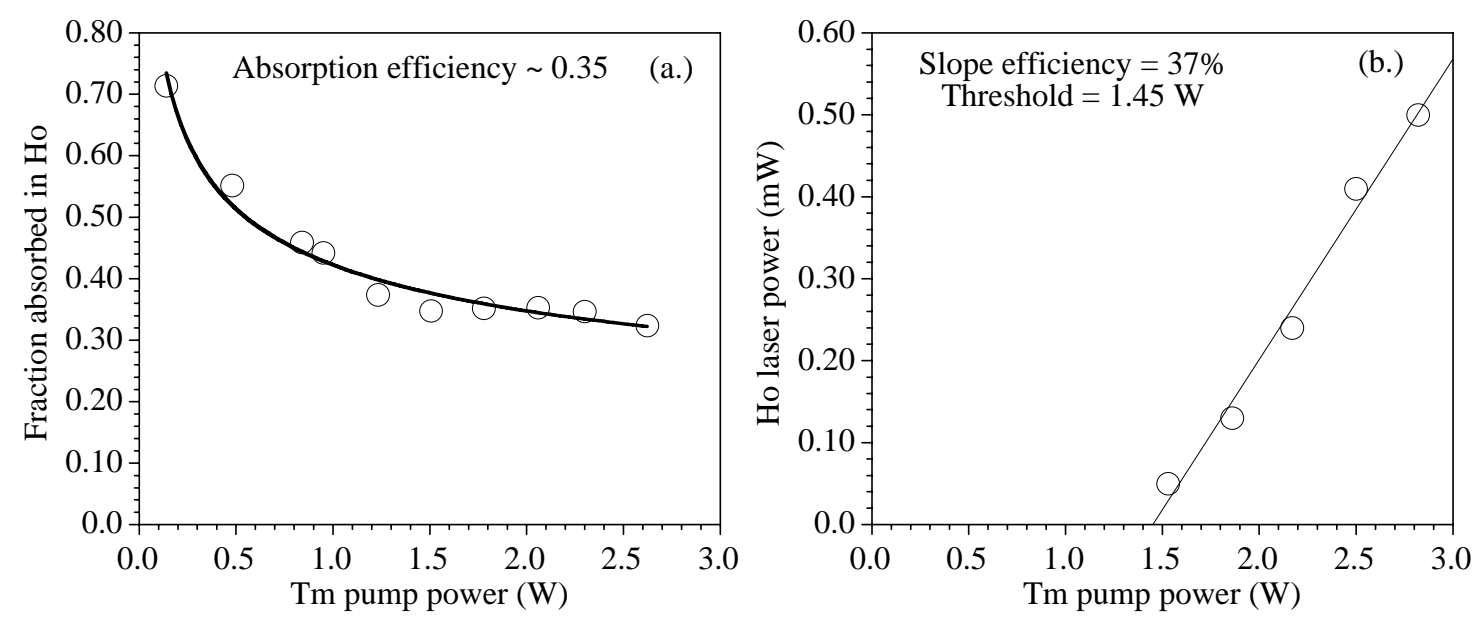

Figure 10. Schematic of a Tm:fiber pumped Ho:YAG Laser.

A nominal concentration of $1.0 \%$ Ho was present in the samples used for these experiments. The Ho:YAG rod length was $8 \mathrm{~mm}$ and the output reflectivity was 0.8 . Pump beam radius, laser beam radius and output mirror reflectivity were systematically studied to achieve slope efficiencies commensurate with the absorption efficiency. [35] 


\subsection{Tm pumping Ho - Literature Review}

Finally, table 1 collects some relevant information on Tm pumped Ho lasers in the literature [34-45]. In these studies, Tm:YALO, Tm:YLF and Tm:fiber pumps have been used. The Tm pump wavelength appears in column 2. The mode (cw or pulsed) appears in column 3. The Ho laser appears in column 4, with the concentration of Ho ions given. The laser material length appears in column 5 . The output mirror reflectivity, slope efficiency and threshold appear in columns 6, 7, and 8, respectively. The reference number for the publication is in column 9, and ordered chronologically from top (earliest) to bottom.

Table 1. Comparison of Tm pumping Ho lasers in the literature.

\begin{tabular}{|l|l|l|l|r|l|l|r|r|}
\hline Pump & $\lambda_{\mathrm{p}}(\mu \mathrm{m})$ & mode & Ho laser & $l(\mathrm{~mm})$ & $\mathrm{R}_{\mathrm{m}}$ & $\sigma_{\mathrm{s}}$ & Threshhold & Ref. \\
\hline Tm:YAlO & 1.9 & cw & YAG & 8 & 0.90 & 0.47 & $2.2 \mathrm{~W}$ & {$[36]$} \\
\hline Tm:YAlO & 1.94 & pulsed & $2.0 \%$ YLF & 10 & 0.87 & 0.20 & $25 \mathrm{~mJ}$ & {$[37]$} \\
\hline Tm:YLF & 1.907 & cw & $2.0 \%$ YAG & 20 & 0.85 & 0.59 & $3.5 \mathrm{~W}$ & {$[38]$} \\
\hline Tm:Fiber & 1.906 & cw & $1.0 \%$ YAG & 8 & 0.80 & 0.37 & $1.45 \mathrm{~W}$ & {$[35]$} \\
\hline Tm:YLF & 1.94 & cW & $0.5 \%$ YLF & 40 & 0.85 & 0.54 & $9.0 \mathrm{~W}$ & {$[39]$} \\
\hline Tm:YLF & 1.9 & quasi- & $0.5 \%$ YAG & 35 & 0.50 & 0.19 & $100 \mathrm{~mJ}$ at & {$[40]$} \\
& & cW & & & & & $60 \mathrm{~Hz}$ & \\
\hline Tm:Fiber & 1.906 & cW & $1.0 \%$ YAG & 20 & 0.90 & 0.67 & $0.6 \mathrm{~W}$ & {$[41]$} \\
\hline Tm:Fiber & 1.906 & cW & $2.0 \%$ YAG & 10 & 0.90 & 0.47 & $1.0 \mathrm{~W}$ & {$[42]$} \\
\hline Tm:YLF & 1.907 & pulsed & $1.0 \%$ YAG & 16 & 0.96 & 0.41 & $3.28 \mathrm{~mJ}$ & {$[34]$} \\
\hline Tm:Fiber & 1.94 & cW & $0.5 \%$ YLF & 40 & 0.30 & 0.42 & $12 \mathrm{~W}$ & {$[43]$} \\
\hline Tm:Fiber & 1.908 & cW & $1.6 \%$ YAG & 0.5 & 0.96 & 0.42 & $9.4 \mathrm{~W}$ & {$[44]$} \\
\hline Tm:Fiber & 1.941 & cw & $1.0 \%$ YAG & 30 & 0.60 & 0.55 & $5.36 \mathrm{~W}$ & {$[45]$} \\
\hline
\end{tabular}

\section{Summary}

A review of Tm and Ho materials has been presented. The applications of $2-\mu \mathrm{m}$ lasers have been discussed and a brief account the historical development has been given. The concept of quasi-four level lasers has been introduced and compared to three level and four level lasers. The challenges of Tm and Ho lasers have been discussed in this context. Energy transfer processes in singly doped Tm and Ho materials as well as Tm:Ho co-doped materials has been presented. The later illustrates a complex set of interactions, not all of which are advantageous for $2-\mu \mathrm{m}$ lasers. The identification of energy transfer processes aids in laser modeling. A rate equation approach that includes 
the laser dynamics, energy transfer and decay processes is a useful tool for simulating laser performance. The fundamental aspects of modeling have been covered in some detail. Finally, recent advances in Tm and Ho materials have been discussed. The concept of using Tm lasers to pump Ho lasers have some advantageous over conventional Tm:Ho lasers. Separating the Tm and Ho atoms minimizes the deleterious upconversion and eliminates Ho to Tm backtransfer. Two experimental approaches have been presented and a survey of research in the literature over the last decade has been provided.

The intention here has not been to produce a complete review, as this endeavor would certainly fill a book. The main concepts and fundamental aspects of these laser materials have been presented as a sort of primer. It is hoped that the concepts covered here and the selected references provide sufficient material for further study. Despite the large amount of work that has been done on Tm and Ho materials, these materials continue to be of interest in the field of lanthanide spectroscopy and lasers.

\section{References}

1. Hale, G. M., Querry, M. R., 1973, Appl. Opt.,12, 555.

2. Barnes, N.P., Filer, E.D., Naranjo, F.L., Rodriquez, W.J., Kokta, M.R., 1993, Opt. Lett., 18, 708.

3. Filer, E.D., Morrison, C.A., Barnes, N.P., Walsh, B.M., 1994, Proceedings on Advanced Solid State Lasers, 20, 127.

4. Johnson, L.F., Boyd, G.D., Nassau, K., 1962, Proc. IRE, 50, 86.

5. Johnson, L.F., Boyd, G.D., Nassau, K., 1962, Proc. IRE, 50, 87.

6. Taylor, Nick, 2000, LASER: The inventor, the Nobel laureate, and the thirty-year patent war (New York: Simon \& Schuster).

7. Johnson, L.F., Geusic, J.E., Van Uitert, L.G., 1966, Appl. Phys. Lett., 8, 200.

8. Chicklis, E.P., Naiman, C.S., Folweiler, 1971, Appl. Phys. Lett., 19, 119.

9. Duczynski, E.W., Huber, G., Ostroumov, V.G., Shcherbakov, I.A., 1986, Appl. Phys. Lett., 1562.

10. Allen, R., Esterowitz, L., Goldberg, L., Weller, J.F., Storm, M. 1986, Tunable Solid State Lasers, 9, 144.

11. Kintz, G., Esterowitz, L., Allen, R., 1987, Tunable Solid State Lasers, 20, 20.

12. Henderson, S.W., Hale, C.P., Magee, J.R., Kavaya, M.J., Huffaker, A.V., 1991, Opt. Lett., 16, 773.

13. Yu, J, Trieu, B.C., Modlin, E.A., Singh, U.N., Kavaya, M.J., Chen, S., Bai, Y., Petzar, P.J., Petros, M., 2006, Opt. Lett., 31, 462.

14. Barnes, N.P., Rodriguez, W.J., Walsh, B.M., 1996, J. Opt. Soc. Am. B, 13, 2872.

15. Förster, T., 1948, Ann. Phys., 21, 55.

16. Dexter, D.L., 1953, J. Chem. Phys., 21, 836.

17. Barnes, N.P., Walsh, B.M., Filer, E.D., 2003, J. Opt. Soc. Am. B, 20, 1212.

18. Antipenko, B.M., Glebov, A.S., Kiseleva, T.I., Pismennyi, V.A., 1986, Opt. Spektrosk., 60, 153. 
19. Armagan, G., Walsh, B.M., Barnes, N.P., Modlin, E.A., Buoncristiani, A.M., 1994, Proceedings on Advanced Solid State Lasers, 20, 141.

20. Tkachuk, A.M., Joubert, M.F., Moncorge, R., Mironov, D.I., Nikitichev, A.A., 1998, Opt. Spectrosc., 85, 885.

21. Walsh, B.M., Barnes, N.P., Di Bartolo, B., 1997, J. Lumin., 75, 89.

22. Walsh, B.M., Barnes, N.P., Di Bartolo, B., 2000, J. Lumin., 90, 39.

23. Kim, K.H., Choi, Y.S., Hess, R.V., Bair, C.H., Brockman, P., Barnes, N.P., Grew, G.W., Kokta, M.R., 1990, Advanced Solid State Lasers, 34, 155.

24. Petrin, R.R., Jani, M.G., Powell, R.C., 1992, Opt. Mat., 1, 111.

25. Barnes, N.P., Filer, E.D., Morrison, C.A., Lee, C.J., 1996, IEEE J. Quant. Elec., 32, 92.

26. Rustad, G., Stenersen, K., 1996, IEEE J. Quant. Elec., 32, 1645.

27. Sousa, J.M., J.R. Salcedo, J.R., Kuzmin, V.V., 1997, Appl. Phys. B, 64, 25.

28. Bruneau, D., Delmonte, S., Pelon, J., 1998, Appl. Opt., 37, 8406.

29. Bourdet, G.L., Lescroart, G., 1999, Appl. Opt., 38, 3275.

30. Walsh, B.M., Barnes, N.P., Petros, M., Yu, J., Singh, U.N., 2004, J. Appl. Phys., 95, 3255.

31. Louchev, O.A., Urata, Y., Saito, N., Wada, S., 2007, Opt. Express, 15, 3940.

32. Louchev, O.A., Urata, Y., Saito, N., Wada, S., 2007, Opt. Express, 15, 11903.

33. Barnes, N.P., Murray, K.E., Jani, M.G., 1997, Appl. Opt., 36, 3363.

34. Barnes, N.P., Reichle, D,J., Walsh, B.M., 2005, Tech. Digest Advanced Solid State Photonics, paper TuB13.

35. Barnes, N.P., Clarkson,W.A., Hanna, D.C., Matera, V., Walsh, B.M., 2001, Technical Digest Conference on Lasers and Electro-optics, 530.

36. Budni, P.A., Pomeranz, L.A., Miller, C.A., Dygan, B.K., Lemons, M.L., Chicklis, E.P., 1998, Advanced Solid State Lasers, 19, 204.

37. Petros, M., Yu, J., Singh, U.N., Barnes, N.P., 2000, Advanced Solid State Lasers, 34, 178.

38. Budni, P.A., Lemons, M.L., Mosto, J.R., Chicklis, E.P., 2000, IEEE J. Quant. Elec., 6, 629.

39. Dergachev, A., Moulton, P.F., 2003, Advanced Solid State Photonics, 83, 137.

40. Budni, P.A., Ibach, C.R., Seltzer, S.D., Gustafson, E.J., Castro, R.T., Chicklis, E.P., Opt. Lett., 28, 1016.

41. Shen, D.Y., Abdolvand, Cooper, L.J., Clarkson, W.A., 2004, Appl. Phys. B, 79, 559.

42. Shen, D.Y., Clarkson, W.A., Cooper, L.J., Williams, R.B., 2004, Opt. Lett., 29, 2396.

43. Dergachev, A., Moulton, P.F., Drake, T.E., 2005, Advanced Solid State Photonics, 98, 608.

44. Schellhorn, M., 2006, Appl. Phys. B, 85, 549.

45. Bai, Y., Petros, M., Yu, J., Petzar, P., Trieu, B., Chen, S. Lee, H., Singh, U., 2006, Tech. Digest Advanced Solid State Photonics, paper TuB7.

\section{B.M. Walsh}

NASA Langley Research Center, Hampton, VA USA 
Office ph: 757-864-7112

Fax: 757-864-8828

Home ph: 757-868-8672

e-mail: brian.m.walsh@nasa.gov 